\title{
Abolition of cyclic activity changes following amygdaloid lesions in rats
}

\author{
STEVEN G. BARTA, ERNEST D. KEMBLE, and ERIC KLINGER \\ University of Minnesota, Morris, Minnesota 56267
}

\begin{abstract}
During extinction of a runway response, the activity levels of normal rats rise above, fall below, and finally return to baseline values in a regular sequence. In the present experiment amygdaloid lesions abolished these sequential activity changes. The results suggest that amygdaloid lesions abolish both the frustrational and depressional components of experimental extinction.
\end{abstract}

During extinction of a runway response, the activity levels of normal rats first rise above, then drop below, and finally return to baseline (Klinger, Barta, \& Kemble, 1974). The demonstration of these cyclic activity changes was stimulated by a more general theory of incentive disengagement (Klinger, in press) in which frustration is followed by response invigoration and then depression (decreased response vigor) which, in turn, is terminated by recovery to baseline. If these cyclic activity changes reflect underlying emotional/motivational processes, they might be expected to respond to manipulations which are known to alter emotional reactivity. Amygdaloid lesions are known to produce a striking reduction in emotional responsiveness in a variety of species and under a wide variety of treatments (e.g., Gloor, 1960; Goddard, 1964). Recently, Henke and Maxwell (1973) have also shown that amygdaloid damage abolishes the frustration produced reactions of rats in a runway. Thus, it seemed of interest to explore the effects of amygdaloid damage on the cyclic activity changes which accompany experimental extinction.

\section{METHOD}

\section{Subjects}

The subjects were 23 male hooded rats (Simonsen Laboratories), weighing $305-400 \mathrm{~g}$ at the time of surgery. All the subjects had previously served in two experiments (Barta \& Kemble, in preparation; Klinger, Barta, \& Kemble, 1974).

Amygdaloid group $(\mathbf{N}=10)$. The subjects had previously acquired a runway response (48 trials) which had then been extinguished (16 trials), and had received five 1-min activity tests. Subsequently, the subjects had sustained amygdaloid lesions and runway performance had been restabilized (80 trials.

Extinction control group $(\mathbf{N}=9)$. As above, except that the surgery consisted entirely of control procedures (see below).

Nonextinction control group $(\mathbf{N}=4)$. The subjects had previously only received 64 rewarded runway trials and five 1-min activity trials.

This research was supported in part by a grant-in-aid from the University of Minnesota Graduate School.

Requests for reprints should be sent to Steven G. Barta, Division of Social Sciences, University of Minnesota, Morris, Minnesota 56267 .

\section{Surgery and Histology}

Surgical and histological procedures are described in previous publications (e.g., Kemble \& Beckman, 1970). Briefly, amygdaloid lesions $(\mathrm{N}=10)$ were produced by passing $2.0-\mathrm{mA}$ anodal dc through the uninsulated tip of a stainless steel pin for $20 \mathrm{sec}$. Operated control subjects $(\mathrm{N}=3)$ received identical treatment but no current passage and the remaining $(\mathrm{N}=6)$ subjects received scalp incisions only.

At the conclusion of testing, 24-micron coronal sections were prepared through all lesions.

\section{Apparatus}

Training was carried out in a Hunter (Model 380) straight runway measuring $10 \times 10 \times 76 \mathrm{~cm}$. Latencies were measured from the startbox door to a photocell relay $76 \mathrm{~mm}$ from the goalbox door. Activity levels were measured in a flat black activity box $(81 \times 81 \times 41 \mathrm{~cm})$ whose floor was divided into $40-\mathrm{cm}$ squares by thin white lines.

\section{Procedure}

Body weight was maintained at $85 \%( \pm 2 \%)$ of its ad-lib value throughout this experiment. The runway performance of all subjects was stabilized by 12 food-rewarded runway trials ( 4 trials/day, intertrial intervals of at least $15 \mathrm{~min}$, reward of five $45-\mathrm{mg}$ Noyes food pellets). Amygdaloid $(\mathrm{N}=10)$ and extinction control $(\mathrm{N}=9)$ groups then received 16 nonrewarded trials. Nonextinction control subjects $(\mathrm{N}=4)$ received an additional 16 rewarded trials. Following the 10th runway stabilization trial, all the subjects received a single 1-min test in the activity box. Number of squares entered during this test served as baseline activity levels. During extinction, all subjects received 1-min activity tests following every second runway trial.

\section{RESULTS}

All lesions included bilateral damage to the amygdala. These lesions tended to be centered about the ventromedial quadrant of this structure with consistent damage in the area of the cortical, medial central and basomedial nuclei as well as the pyriform cortex. Damage to the optic tracts $(\mathrm{N}=4)$ was sometimes noted but was not related to runway performance or activity levels. Figure 1 depicts four representative lesions.

All subjects rapidly reacquired the runway response, with latencies decreasing markedly over trials $[F(6,120)$ $=18.43, \mathrm{p}<.005]$. During restablization there were no differences in runway performance among groups $[F(2,20)=.19]$ and no Groups by Trials interaction $[F(12,120)=1.25]$. During extinction the runway 


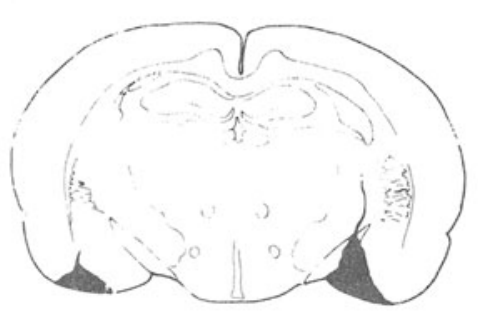

$S * 15$

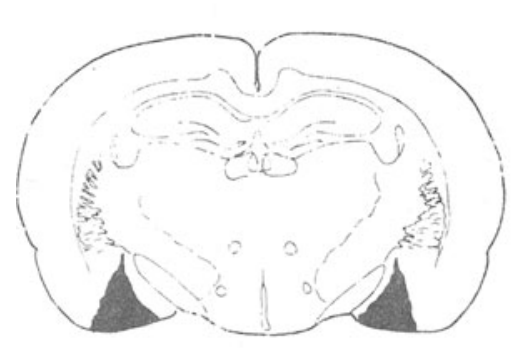

$\mathrm{S} \# 22$

latencies of the amygdaloid and extinction control groups increased sharply $[\mathrm{F}(4,68)=17.97, \mathrm{p}<.001]$. The nonextinction control group continued to perform at a steady rate and by the end of extinction ran significantly more rapidly than either amygdaloid $[\mathrm{U}(4,10)=0, \mathrm{p}<.001]$ or extinction control subjects $[\mathrm{U}(4,9)=1, \mathrm{p}<.01]$.

The activity levels of all groups during this experiment are depicted in Figure 2. It can be seen that the activity levels of all groups were quite similar during restabilization trials with no suggestion of group differences, $p>.10$. During extinction trials, the extinction control group showed the expected upswing, downswing, and recovery to baseline [overall trials $\mathrm{F}(8,64=6.74, \mathrm{p}<.005]$. The activity levels of amygdaloid and nonextinction control groups showed no systematic change over trials $[F(8,72)=1.14$ and $\mathrm{F}(8,24)=1.10$, respectively $]$. The activity levels of these two groups also did not differ significantly from each other $[F(1,12)=.07]$.

Extended trend analysis of variance of activity levels revealed a strong cubic orthogonal component of the extinction control group's trend $[\mathrm{F}(1,64)=24.54$, $\mathrm{p}<.001]$, and signficant linear $[\mathrm{F}(1,64)=17.56$, $\mathrm{p}<.001]$ and quadratic $[\mathrm{F}(1,64)=6.50, \mathrm{p}<.025]$ orthogonal components. The post-runway activity levels of the amygdaloid and nonextinction control groups contained no significant orthogonal components. The Groups by Trials interaction comparing amygdaloid and extinction control subjects contained a highly significant cubic component $[F(1,17)=49.42, p<.001]$, and the Groups by Trials interaction comparing all groups also contained a significant cubic orthogonal component
Figure 1. Four representative amygdaloid lesions at the level of greatest destruction.

$[F(1,20)=33.11, \mathrm{p}<.001]$

For extinction control subjects, the mean activity levels following runway extinction Trials 4 and 6 (analyzed by Duncan's multiple range test) are significantly higher than all others, $\mathrm{p}<.001$. The mean activity levels following Trials 10 and 12 are not significantly lower than those on the baseline trial and following Trials 14 and 16 (recovery) contrary to prediction from earlier data (Klinger, Barta, \& Kemble, 1974), but 7 of 9 animals were more active following Trials 14 and 16 (combined) than following Trials 10 and 12 (binomial $p=.08$ ). The extinction control group's mean activity levels were not significantly lower than the amygdaloid group's activity following Trials 10 and 12 .

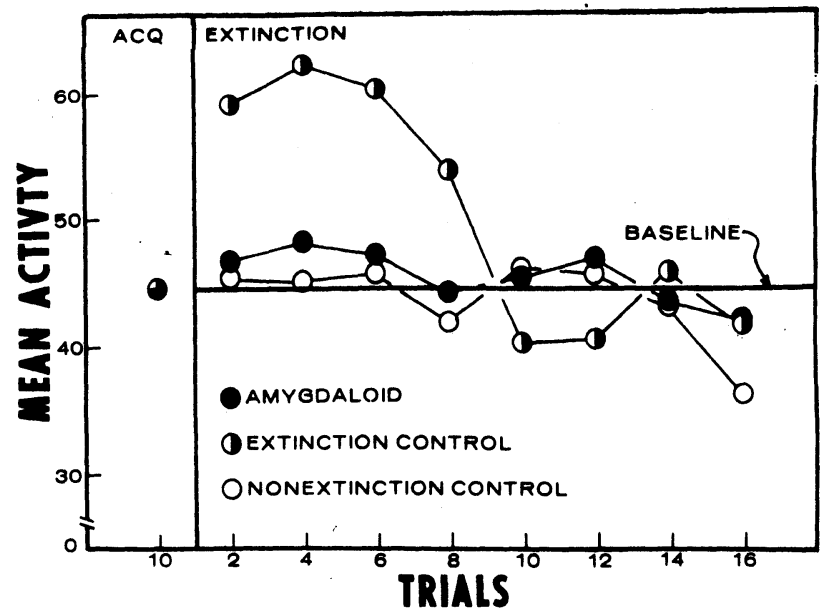

Figure 2. Activity levels during acquisition (ACQ) and extinction of runway response. Numbers on abscissa refer to runway trials. 


\section{DISCUSSION}

The activity levels of extinction control subjects showed a clear invigoration, depression, and return to baseline in the present experiment. These activity changes were very similar to those noted in the original experiment (Klinger, Barta, \& Kemble, 1974) and indicate that such cyclic activity changes are a replicable phenomenon during extinction. As before, these changes bore no discernible consistent relation to runway running speeds.

The data also clearly indicate that amygdaloid lesions abolish both the invigoration and depression of activity levels during extinction. The activity levels of these subjects were indistinguishable from those of subjects that were not extinguished. These data extend an earlier report (Henke \& Maxwell, 1973) that amygdaloid lesions abolish frustrative response invigoration. In addition, the data suggest that these lesions also abolish the depressive reactions which follow invigoration during extinction. Such a suggestion is consistent with the rather sweeping attenuation in emotional reactivity which follows these lesions (e.g., Schreiner \& Kling, 1956). The fact that both the invigoration and depression phases of the activity cycle are eliminated by amygdaloid lesions supports (though not conclusively) the conception that the cycle is mediated by closely related mechanisms.

\section{REFERENCES}

Gloor, P. Amygdala, In J. Field (Ed.), Handbook of physiology. Vol. 2, Neurophysiology. Washington: American Physiological Society, 1960, Pp. 1395-1420.

Goddard, G. V. Functions of the amygdala. Psychological Bulletin, 1964, 62, 89-106.

Henke, P. G., \& Maxwell, D. Lesions in the amygdala and the frustration effect. Physiology and Behavior, 1973, 10, 647-650.

Kemble, E. D., \& Beckman, G. J. Runway performance of rats following amygdaloid lesions. Physiology and Behavior, 1970, $5,45-47$

Klinger, E. Consequences of commitment to and disengagement from incentives. Psychological Review, in press.

Klinger, E., Barta, S. G., \& Kemble, E. D. Cyclic activity changes during extinction in rats: A potential model of depression. Animal Learning \& Behavior, 1974, 2, 313-316.

Schreiner, L., \& Kling, A. Rhinencephalon and behavior. American Journal of Physiology, 1956, 184, 486-490.

(Received for publication November 11, 1974.)

\title{
Comparable ratio indices of sequential consistency and subjective clustering in multitrial free recall
}

\author{
JOHN B. TODMAN \\ City of Leicester College of Education, Leicester, LE 7 9SU, England
}

\begin{abstract}
Structurally comparable indices of clustering and sequential consistency are described. The former is the ratio index of subjective clustering, and the latter is the ratio index of repetition. Both indices are applicable to the same set of multitrial free-recall protocols, and it is suggested that they may be helpful in clarifying issues arising from contradictory results obtained in studies employing different free-recall paradigms. Correlational limitations of the indices are discussed.
\end{abstract}

Organization in the recall of "unrelated" word lists has generally been quantified in terms of the consistency of recall sequences on successive trials, following different random orders of presentation of the list items. The quantification of organization in the recall of categorized lists, on the other hand, has usually been based on the extent of clustering of category items in

This paper is sponsored by Colin V. Newman, who takes full editorial responsibility for it. John $\dot{B}$. Todman is now at the Department of Psychology, University of Dundee, Dundee DD1 4HN, Scotland from March 1, 1975. The author wishes to thank A. W. MacRae for checking the derivations of formulae and $T$. Teasdale for providing a program to compute the organization indices. the recall protocol following a single presentation :rial. It is frequently assumed (e.g., Shuell, 1969) that the same basic psychological processes are being tapped in these different paradigms, yet contradictory results have been obtained in developmental studies, depending upon whether an index of sequential consistency or a clustering index has been used. Laurence (1966) found no increase in sequential consistency with age, even though recall increased with age from 6 to 10 years, whereas other (e.g., Vaughan, 1968) have found both clustering and recall to increase with age. Discrepancies of this sort could be due to differences in the underlying probability models, or peculiarities of the mathematical 